\title{
Genom-Engineering in der wissenschaftlichen und klinischen Praxis
}

Andreas Reinisch, befragt von Johann Platzer

Genom-Engineering gehört zu den vielversprechendsten biomedizinischen Entdeckungen. Die rasante Entwicklung auf dem Gebiet der Gentechnik wirft aber auch eine Vielzahl wissenschaftlicher, rechtlicher und ethischer Fragen auf. Andreas Reinisch ist Hämatologe und forschte unter anderem in den Vereinigten Staaten an der Stanford University. In einem Interview mit Johann Platzer gibt er Einblicke in seine praktische Arbeit. Er spricht über verschiedene Anwendungsbereiche von Gentechnik sowie über Zukunftsperspektiven und mögliche Grenzen. Der Wissenschaftler glaubt, dass auch in Zukunft Eingriffe in die Keimbahn weiterhin tabu sein werden - die Grenzen zwischen genetischen Therapien und Genom-Engineering zu Enhancement-Zwecken werden sich jedoch weiterhin verschieben.

Platzer: Herr Dr. Reinisch, Sie arbeiten in der Klinischen Abteilung für Hämatologie der Medizinischen Universität Graz und sind Experte für Genom-Engineering. Was versteht man konkret darunter?

Reinisch: $\quad$ Unter Genom-Engineering - häufig auch Genome-Editing genannt - versteht man die gezielte Veränderung der DNA mittels unterschiedlicher molekularbiologischer Methoden. Dabei kann man bestimmte Gene ausschalten, neue Gene einbringen oder Gene korrigieren.

Platzer: $\quad$ In welchen Bereichen arbeiten Sie konkret?

Reinisch: Ich bin Hämatologe und beschäftige mich hauptsächlich mit der Entstehung von Blutkrebserkrankungen. Wir verwenden Genom-Engineering, um unterschiedliche Mutationen, die normalerweise Blutkrebserkrankungen auslösen, nachzubilden und somit deren genauen Funktion zu untersuchen. Im Rahmen meines Auslandsaufenthaltes in den USA habe ich auch an der „Sichelzell-Erkrankung“ gearbeitet. Das ist eine vererbbare monogenetische Erkrankung der 
roten Blutkörperchen, die mittels Genom-Engineering therapierbar ist.

Platzer: Durch Eingriffe in die DNA eröffnet sich ja eine Fülle von Anwendungsbereichen. Welche Arten des Genom-Engineering lassen sich diesbezüglich allgemein unterscheiden?

Reinisch: Zunächst gibt es hier einmal ein riesiges Forschungsfeld in der Landwirtschaft - konkret in der Tier- und Pflanzenzucht. Es gibt da schon unglaubliche Möglichkeiten, Pflanzen so zu verändern, dass sie widerstandsfähiger oder ertragsreicher werden. Damit könnte man z. B. die Nahrungsknappheit in bestimmten Gebieten der Erde bekämpfen. Man versucht durch Gentechnik aber auch Krankheiten zu bekämpfen, die durch unterschiedliche Parasiten verursacht werden. Bei der Malaria zum Beispiel, die ja durch eine Stechmücke übertragen wird, werden Verfahren getestet, in denen diese Mücken durch einen sogenannte Gene Drive gentechnisch verändert werden. Damit wird es möglich, ein "Sterblichkeits-Gen” in der Anopheles-Population zu verbreiten und so deren Vermehrung zu unterbinden.

Platzer: Und beim Menschen?

Reinisch: $\quad$ Auch hier gibt es schon eine Reihe von möglichen Anwendungsbereichen. Man unterscheidet hier zunächst einmal zwischen der somatischen Therapie und der Keimbahntherapie. Die somatische Gentherapie ist ja allgemein akzeptiert. Eingriffe in die Keimbahntherapie hingegen sind natürlich höchst problematisch, da diese ja zukünftige Generationen betreffen und nicht mehr rückgängig gemacht werden können. Es ist aber auch schon eine Reihe von Verfahren möglich, die in Richtung Enhancement gehen. Hier versucht man dann Eigenschaften oder Fähigkeiten des Menschen ohne medizinische Indikation zu verbessern. 
„Durch unterschiedliche gesetzliche Regelungen in verschiedenen Ländern könnte es leicht zu einem Behandlungstourismus kommen."

Platzer:

Reinisch: $\quad$ In Österreich ist, so wie überall auf der Welt, nur die soma-

Welche Formen von "Genom-Engineering" sind in Österreich zugelassen? tische Gentherapie am Menschen erlaubt. Man braucht dafür aber eine behördliche Genehmigung. Außerdem darf diese Therapie nur von ärztlichem Personal in einer Krankenanstalt durchgeführt werden. Es wird aber in Österreich meines Wissens noch wenig mittels Genom-Engineering therapiert - und ähnlich ist es in ganz Europa. Jedoch wird für die Herstellung einer neuartigen, sehr speziellen Form der Krebstherapie, die sich CAR-T-Zell-Therapie nennt, Genom-Engineering eingesetzt. Diese genetisch veränderten Zellen können bei verschiedenen Tumorerkrankungen eingesetzt werden. Dabei werden verschiedene T-Zellen der Patienten genetisch verändert und dann wieder in diesen Patienten rücktransplantiert. Der Eingriff am Gen passiert aber in spezialisierten Labors. Das heißt, wir schicken die Zellen zunächst nach Amerika. Dort werden sie dann genetisch modifiziert und danach wieder nach Österreich zurückgeschickt - und wir verabreichen sie dann wieder dem Patienten.

Platzer: Wie sieht es international aus?

Reinisch: Wir kennen ja alle den bekannten Fall aus China. Dort hat im Jahr 2018 der Biophysiker Jiankui He sogar die Keimbahn mittels der Genschere CRISPR/Cas9 verändert. Die Keimbahntherapie ist aber auch in China verboten, und Dr. He musste dafür ja ins Gefängnis. Die Therapie von genetisch veränderten somatischen Zellen hingegen ist in China gang und gäbe. Das gilt auch für Amerika. In Europa gelten hingegen strengere Regeln, vor allem was die Zulassung betrifft. Es sind daher erst wenige Gentherapien von der Europäischen Arzneimittelbehörde (EMA) genehmigt. Es laufen hier auch viel weniger Studien. Nichtsdestotrotz wird sie auch bei uns über kurz oder lang zur Standardtherapie gehören. 
Platzer: Die Vereinigten Staaten und China werden immer wieder als „Vorzugsländer" der Genforschung genannt. Glauben Sie, dass Europa hier den Anschluss verlieren könnte?

Reinisch: Der Hauptgrund, warum Europa schon jetzt etwas den Anschluss verliert, liegt darin, dass die Förderungsquoten in Europa einfach sehr niedrig sind und die Wissenschaft hier nicht so massiv unterstützt wird, wie es in China und in den USA üblich ist. Außerdem gehen sehr viele gute Wissenschaftler von Europa nach Amerika und kommen dann nicht mehr zurück. Die Forschung wird in Amerika einfach viel mehr geschätzt - das habe ich selbst erlebt.

Platzer: $\quad$ Könnte hier nicht auch ein gewisser Druck entstehen, bestimmte umstrittene Verfahren rascher zuzulassen?

Reinisch: Ja, das glaube ich schon - vor allem dann, wenn immer mehr Technologien zur Verfügung stehen und diese aber nicht eingesetzt werden dürfen. Ich spreche hier natürlich vom therapeutischen Bereich. Die Regulative sind diesbezüglich in den USA sicherlich gleich groß wie in Europa. In China ist da meines Wissens vieles einfacher umzusetzen und von der Forschung auf direkterem Weg in die klinische Anwendung zu translatieren. Durch unterschiedliche gesetzliche Regelungen in verschiedenen Ländern kann es durchaus zu einem Behandlungstourismus kommen, vor allem für jene, die es sich leisten können.

\section{„CRISPR/Cas9 war eine biotechnische Revolution."}

Platzer: Kommen wir nun zu den einzelnen Anwendungsbereichen: Welche Fortschritte haben sich in den vergangenen Jahren bezüglich der somatischen Körperzelltherapie ergeben?

Reinisch: Diese Therapieform gibt es ja schon länger. Ich glaube, der größte Fortschritt war eindeutig die Entdeckung von CRISPR/Cas9, also die Entdeckung eines Enzyms, das Bakterien verwenden, um sich sehr gezielt vor Viren zu schützen, indem sie deren Ribonukleinsäuren zerschneiden. Dann hat man Anfang der 2010er Jahre herausgefunden, dass dieses Cas9-Enzym in Kombination mit einem RNAMolekül auch die menschliche DNA mit unglaublicher Prä- 
zision schneiden kann. Das war dann eine biotechnologische Revolution, wie es sie noch nie gegeben hat. CRISPR/ Cas9 ist nämlich extrem schnell verwendbar, unglaublich genau und äußerst effizient. Was zuvor Jahre oder gar Jahrzehnte gedauert hat, kann mittlerweile in Monaten gemacht werden - und das war der große Quantensprung.

Platzer: $\quad$ Welches Potential sehen Sie hier noch für die somatische Therapie?

Reinisch: $\quad$ Ein Problem ist derzeit noch, dass somatische Gentherapie in vielen Fällen noch Viren (Lenti-Adeno- und/oder Adenoassoziierte Viren) benötigen, damit man Reagenzien in die Zellen einbringen kann. Das dürfte aber immer weniger werden, und das ist positiv. Das zweite Problem ist, dass CRISPR/Cas9 Doppelstrangbrüche in der DNA erzeugt. Diese Doppelstrangbrüche können aber auch negative Folgen für die Zellen haben. Es gibt mittlerweile aber auch schon neue Methoden, wo man Gene verändern kann ohne Doppelstrangbrüche zu verursachen. Da besteht meines Erachtens noch großes Potential. Ein weiteres Problem liegt darin, dass man zukünftig nicht nur monogenetische Erkrankungen wird behandeln können, sondern auch polygenetische - also solche, die durch mehrere Gene ausgelöst werden. Allerdings muss man hier noch vorsichtig sein. Man weiß nämlich vielerorts noch nicht, wie die einzelnen Gene miteinander kommunizieren und welche Konsequenzen diese Komplexität mit sich bringen wird.

\section{"Ein Null-Risiko wird es nie geben."}

Platzer: Kommen wir nun zu möglichen Risken. Wie sicher ist GenomEngineering allgemein?

Reinisch: Risiken gibt es natürlich nach wie vor. CRISPR/Cas9 ist zwar extrem genau und wesentlich schneller als frühere Verfahren, allerdings gibt es bei vielen Gen-Therapien auch sogenannte Off-Target-Effekte. Das heißt, das Enzym, das die DNA durchschneidet, wird zunächst mittels einer „Guide-RNA“ an die DNA herangeführt. Und diese „GuideRNA“ kann ganz spezifische DNA-Stücke binden. Sie findet 
aber leider auch manchmal andere Stellen in der DNA, die dann auch durchgeschnitten werden. Somit kann es auch dort zu unerwünschten genetischen Veränderungen kommen, und das kann dann Probleme auslösen. Das sind natürlich Nebenwirkungen, die wir möglichst vermeiden wollen. Durch die neuen, verbesserten Technologien werden diese Nebenwirkungen aber immer weniger. Das heißt, wir reden hier von 0,1 bis 0,2 Prozent der Schnitte, die dann nicht „on-target“, sondern „off-target“ passieren. Allerdings weiß man noch nicht, wie sich das dann in weiterer Folge auswirken wird, wenn man solche Zellen transplantiert.

Platzer: $\quad$ Gibt es da überhaupt schon Langzeiterfahrungen?

Reinisch: $\quad$ Es gibt einfach noch zu wenig Daten, dass man sagen könnte, die Methodik sei hundertprozentig sicher. Die Effekte werden sich erst zeigen, wenn die ersten Patienten über mehrere Jahre nachbeobachtet werden. Erst dann kann man sehen, ob die ungewollten genetischen Veränderungen irgendwelche Konsequenzen haben. Für mich ist die große Frage eine andere: Wenn ein Patient eine lebensbedrohliche genetische Erkrankung hat, dann wird man wahrscheinlich dieses geringe Risiko in Kauf nehmen. Irgendeine Gefahr kann es jedoch immer geben. Ein Null-Risiko wird es nie geben.

Platzer: Wie können die Risiken begrenzt werden?

Reinisch: Die Risken können insofern begrenzt werden, als man zunächst einmal die besten Reagenzien auswählt. Dann müssten die veränderten Zellen in präklinischen Modellen ausreichend getestet werden. Das geht von der Maus bis hin zum Primaten. Darüber hinaus sollte man das Ganze eher langsam angehen. 
„Wir haben nicht das Recht, für jemanden etwas zu entscheiden, das wir nicht nachvollziehen können."

Platzer: $\quad$ Nun zu den ethischen Aspekten: Welche Anwendungsmethoden sind aus Ihrer Sicht ethisch verantwortbar?

Reinisch: $\quad$ Also für mich ist einmal die somatische Gentherapie ohne Weiteres ethisch verantwortbar. Alles andere ist für mich problematisch.

Platzer: Das heißt, genetische Eingriffe in die Keimbahn und GenomEngineering zu Enhancement-Zwecken würden Sie absolut verbieten?

Reinisch: $\quad$ Eingriffe in die Keimbahn würde ich auf jeden Fall unter allen Umständen verbieten. Das somatische Enhancement würde ich zwar nicht zur Gänze verbieten, aber es müsste für mich immer zu einem gewissen Maße medizinisch vertretbar sein. Allerdings scheiden sich hier natürlich die Geister, was medizinisch vertretbar ist und was nicht. Es gibt ja auch Verfahren, wo es darum geht, durch GenVeränderungen z.B. stärkere Knochen zu bekommen. Das wäre ja für einen Patienten mit Osteoporose gut, jedoch sollte man z. B. bei einem Profi-Boxer eine solche Therapie nicht durchführen. Das müsste man individuell diskutieren. Aber prinzipiell bin ich dem gegenüber eher abneigend eingestellt.

Platzer: $\quad$ Aus welchen Gründen würden Sie persönlich eine Keimbabntherapie kategorisch verbieten?

Reinisch: Weil es zukünftige Generationen betrifft und nicht rückgängig gemacht werden kann. Außerdem kann man die potentiell Betroffenen ja nicht fragen, ob sie das wollen. Das ist meines Erachtens grenzwertig.

Platzer: $\quad$ Es gibt aber auch Gegenmeinungen. Viele sagen, es kommt ja auch sonst den Eltern ein Entscheidungsrecht für ihre Kinder zu, und dabei muss ja das Kindeswohl im Mittelpunkt stehen. Außerdem ließe sich durch eine Nicht-Einwilligungsmöglichkeit des Kindes noch kein Anspruch ableiten, ein genetisch geschädigtes Genom seiner Vorfahren zu erben anstatt eines gesunden und korrigierten Genoms. Was würden Sie jenen erwidern? 
Reinisch: Das ist eine schwierige Frage. Ich würde wahrscheinlich zurückfragen, wer uns eigentlich das Recht gibt, zu sagen, dass jemand mit einer genetischen Beeinträchtigung nicht durchaus auch ein schönes Leben haben kann! Das muss aber wohl jeder selbst entscheiden. Ich glaube aber, wir haben nicht das Recht, für jemanden etwas zu entscheiden, das wir nicht nachvollziehen können.

Platzer: $\quad$ Mit ibrer skeptischen Haltung befinden Sie sich in prominenter Gesellschaft. Der deutsche Philosoph Jürgen Habermas hat dazu im Kontext der PID eingebracht, dass die Realisierung der eigenen Freiheit und der eigenen Identität des Menschen vor allem an die Unverfügbarkeit der eigenen Naturwüchsigkeit gebunden sei. Man könnte hier auch, wie es Peter Strasser einmal formuliert hat, von einem "Recht auf Kreatürlichkeit" des Menschen sprechen. Wie sehen Sie diese Einschätzung bzw. Forderung?

Reinisch: Ich sehe das ähnlich, obwohl ich mich als Biomediziner noch weniger mit diesen philosophischen Fragen auseinandergesetzt habe. Es kann natürlich sein, dass Eltern, deren Kinder eventuell von einer genetischen Erkrankung betroffen sein könnten, dies anders sehen - und das ist auch nachvollziehbar -, aber aus meiner aktuellen Sichtweise heraus bin ich dagegen.

\section{„Man muss auch viel mehr an die Moral aller Wissenschaftler appellieren."}

Platzer: Bleiben wir bei der Diskussion über etwaige Eingriffe in die Keimbahn: Der deutsche Ethikrat hat sich 2019 mehrheitlich gegen eine kategorische Unantastbarkeit der menschlichen Keimbahn ausgesprochen. Auch Michael Rosenberger vertritt in seinem Beitrag in diesem Band eine ähnliche Haltung. Er spricht sich vielmehr für „prinzipiell revidierbare ethische Limitierungen" aus und begründet seine Position vor allem in der sich verändernden Folgenabschätzung. Welche Position vertreten Sie diesbezüglich?

Reinisch: Dem könnte ich sogar zustimmen. Meine derzeitige Skepsis basiert natürlich auf dem jetzigen Wissen und meiner derzeitigen Sichtweise. Wenn man allerdings zum Beispiel in 
hundert Jahren einmal genau wissen würde, wie alle Gene exakt miteinander vernetzt sind, und dadurch alle Risiken ausschalten könnte, dann könnte man vielleicht darüber nachdenken. Aber aktuell weiß man einfach viel zu wenig darüber. Deshalb ist es meiner Meinung nach momentan nicht vertretbar. Allerdings merke ich gerade, dass ich mich soeben selbst irgendwie widersprochen habe. Ich hatte ja vorher mit Nachdruck gesagt, dass wir nicht das Recht haben, über zukünftige Generationen zu verfügen. Da sieht man, wie komplex diese ganze Thematik ist.

Platzer: Wissenschaftler fordern in diesem Kontext immer wieder weltweite "Moratorien“, also zeitlich befristete Verbote, um reflektierte ethische Auseinandersetzungen zu ermöglichen. Was halten Sie davon und wie hoch schätzen Sie eine solche Durchsetzbarkeit ein?

Reinisch: Da bin absolut dafür, dass man Verbote auf einer höheren Ebene regelt - zumindest europaweit, idealerweise weltweit. Aber die große Frage dabei ist natürlich, wer das kontrollieren soll. Ein Hauptproblem dabei ist immer, dass es besonders attraktiv ist, bei wissenschaftlichen Entdeckungen der Erste zu sein. Denken Sie an Dr. He. Er hat für seine „Aktion“ ja eine Publicity bekommen, die seinesgleichen sucht. Das heißt, es wird letztlich nicht komplett kontrollierbar sein. Die Einhaltung der Regeln kann meines Erachtens nur insofern umgesetzt werden, indem man diejenigen, die sich nicht daran halten, bestraft. Darüber hinaus ist es auch üblich, dass sich die großen wissenschaftlichen Zeitschriften, wie etwa Nature, Cell oder Science, weigern, so etwas zu publizieren. Es gibt nach wie vor keine Publikation über den Eingriff von Dr. He in die Keimbahn. Ich glaube, so sollte es auch weiterhin gehandhabt werden. Man muss auch viel mehr an die Moral aller Wissenschaftler appellieren.

Platzer: Was meinen Sie damit?

Reinisch: $\quad$ Es müsste hier auch ein verstärktes „Peer-Review“ von den Forschenden selbst geben. Dr. He hat hier sicherlich einiges dazu beigetragen, dass man da unter den Wissenschaftlern in Zukunft etwas vorsichtiger sein wird. Dr. He ist ja schon vor einigen Jahren sehr offen mit seinen präliminären Forschungsergebnissen und Absichten umgegangen. Er hatte 
immer wieder angekündigt, dass er einen Eingriff in die Keimbahn vornehmen werde. Seine damaligen Kollegen haben ihn zwar davor abgeraten, aber so richtig ernst genommen haben sie ihn damals nicht. Sie sind damit auch nicht an die Öffentlichkeit getreten. Diesen Wissenschaftlern ist dann später sowohl von den Medien als auch von der wissenschaftlichen Community vorgeworfen worden, dass sie nicht früh genug eingeschritten seien. Auch seinem ehemaligen Betreuer hat man mit negativen Konsequenzen von Seiten der Universität gedroht. Daraus hat man, glaube ich, gelernt, dass man - wenn man so etwas weiß und trotzdem schweigt - sich dadurch mitverantwortlich macht. Wenn ich z. B. jetzt jemanden kennen würde, der behauptet: „Ich mache jetzt einen Keimbahneingriff und ein ,Designerbaby', weil es technisch leicht machbar ist", dann würde ich ihm nicht nur davon abraten, sondern ich würde auch an die wissenschaftliche Community mit dem Appell herantreten, dass wir das verhindern müssen. Da hat mittlerweile ein Umdenken stattgefunden.

Platzer: Kommen wir abschließend noch einmal zum Thema GenomEngineering zu Enhancement-Zwecken: Lässt sich eine Grenze zwischen der Therapie von Krankheiten und der nicht-therapeutischen Steigerung von einzelnen Eigenschaften oder Fähigkeiten überhaupt klar ziehen?

Reinisch: Ich glaube nicht. Diese Grenze wird sich immer wieder verschieben und verändern, weil man über die unterschiedlichen Gene mehr lernen wird und man dann auch über mögliche Verschiebungen von Grenzen diskutieren wird müssen.

Platzer: Kürzlich hat ein bekannter österreichischer Genetiker ein sogenanntes Schlankheitsgen entdeckt. „Die Hemmung des Gens könnte", so der Forscher, "womöglich eine neue Therapiemöglichkeit sein, um schlank zu bleiben." Wo müsste man hier die Grenze zwischen Therapie - zum Beispiel bei Adipositas-PatientInnen - und dem Wunsch nach einem dem gesellschaftlichen Schönheitsideal entsprechenden, schlanken Körper ziehen?

Reinisch: Ich glaube, die Grenzen sollten für alle somatischen Veränderungen dieselben bleiben. Ist es medizinisch indiziert, dann sollte es durchaus diskutiert werden. Dient die genetische Manipulation hingegen nur dem Enhancement, und 
als solches würde ich auch das Ideal des schlanken Köpers sehen, muss man dem Ganzen klare Grenzen setzen. Da dieses spezielle Schlankheitsgen allerdings im Hypothalamus (Gehirn) ausgeschaltet werden muss, wird sich für uns diese Frage noch lange nicht stellen, da wir leider aktuell noch keine effizienten Methoden haben, Nervenzellen in vivo zu manipulieren.

\section{„Genom-Engineering wird in Zukunft zu unserem therapeutischen Standardrepertoire gehören."}

Platzer: $\quad$ Kommen wir zu einer Frage, die eher in Richtung „Pro-Enhancement" durch Genom-Engineering zielt: Wie bereits angesprochen, greift der Mensch ja auch in die Pflanzen- und Tierzucht gezielt durch genetische Veränderungen ein. Warum sollte das beim Menschen verboten sein? Besteht die Kultur des Menschen nicht gerade darin, die Natur gezielt zu verändern?

Reinisch: Das ist eine sehr interessante, aber auch schwierige Frage. Einerseits stimmt das natürlich - vor allem, wenn es um das eigene Überleben geht. Es haben sich ja im Laufe der Evolution auch die überlebensfähigsten Lebewesen durchgesetzt. Anderseits ist dagegen auch so manches einzuwenden: Sobald eine solche Möglichkeit nicht für alle gegeben wäre, ginge das in Richtung Eugenik. Dann hätten einige massive Vorteile gegenüber anderen. Das wäre ungerecht und würde die Gesellschaft massiv zum Negativen verändern.

Platzer: $\quad$ Abschließend noch ein Ausblick: Welche Bedeutung wird Ibres Erachtens Genom-Engineering in etwa zehn Jahren haben?

Reinisch: Ich glaube, dass viele monogenetische Erkrankungen in zehn Jahren durch Genom-Engineering therapiert werden können. Die somatische Gentherapie wird also in Zukunft sicherlich zu unserem therapeutischen Standardrepertoire gehören. Das wird dann zwar nicht jedes kleine Krankenhaus machen können, aber es wird Zentren geben, die somatische Gentherapien standardmäßig anbieten.

Bei der Keimbahntherapie wird sich in den nächsten Jahren nichts verändern, weil sowohl die wissenschaftliche Community als auch die Gesellschaft an sich die eindeutige Mei- 
nung vertritt, dass ein Eingriff in die Keimbahn ein Tabu für uns Menschen darstellt, und deshalb dieses Verbot nicht gelockert werden darf.

Platzer: $\quad$ Herr Dr. Reinisch, herzlichen Dank für das Gespräch! 